\title{
Multi-scale Analysis of Bone Chemistry, Morphology and Mechanics in the oim Model of Osteogenesis Imperfecta
}

\author{
Zachary R. Bart ${ }^{\mathrm{a}}$ \\ Max A. Hammond \\ Joseph M. Wallace ${ }^{\mathrm{a}, c^{\star}}$ \\ ${ }^{a}$ Department of Biomedical Engineering, Indiana University-Purdue University at \\ Indianapolis, Indianapolis, IN, USA \\ ${ }^{b}$ Weldon School of Biomedical Engineering, Purdue University, West Lafayette, IN, USA \\ ${ }^{c}$ Department of Orthopaedic Surgery, Indiana University School of Medicine, \\ Indianapolis, IN, USA
}

\author{
*Corresponding Author: \\ Dr. Joseph M. Wallace \\ Indiana University-Purdue University at Indianapolis \\ Department of Biomedical Engineering \\ 723 W Michigan St. SL220D \\ Indianapolis, IN 46202 \\ Phone: (317) 274-2448 \\ Fax: (317) 278-2455 \\ E-mail: jmwalla@iupui.edu
}

Keywords: Atomic force microscopy, Raman Spectroscopy, Reference Point Indentation, MicroCT, Crystallinity, Modulus

This is the author's manuscript of the article published in final edited form as:

Bart ZR, Hammond MA, Wallace JM. Multi-scale analysis of bone chemistry, morphology and mechanics in the oim model of osteogenesis imperfecta. Connect Tissue Res. 2014 Aug 1;55(S1):4-8. Available from: http://dx.doi.org/10.3109/03008207.2014.923860 


\section{ABSTRACT}

Osteogenesis imperfecta is a congenital disease commonly characterized by brittle bones and caused by mutations in the genes encoding Type I collagen, the single most abundant protein produced by the body. The oim model has a natural collagen mutation, converting its heterotrimeric structure (two $\alpha 1$ and one $\alpha 2$ chains) into $\alpha 1$ homotrimers. This mutation in collagen may impact formation of the mineral phase, creating a brittle bone phenotype in this animal. Femurs from male wild type (WT) and homozygous (oim/oim) mice, all at 12 weeks of age, were assessed using assays at multiple length scales with minimal sample processing to ensure a near-physiological state. Atomic force microscopy (AFM) demonstrated detectable differences in the organization of collagen at the nanoscale that may partially contribute to alterations in material and structural behavior obtained through mechanical testing and reference point indentation (RPI). Changes in geometric and chemical structure obtained from $\mu$-Computed Tomography and Raman spectroscopy indicate a smaller bone with reduced trabecular architecture and altered chemical composition. Decreased tissue material properties in oim/oim mice are likely driven by changes in collagen fibril structure, decreasing space available for mineral nucleation and growth, as supported by a reduction in mineral crystallinity. Multi-scale analyses of this nature offer much in assessing how molecular changes compound to create a degraded, brittle bone phenotype.

\section{INTRODUCTION}

The fundamental constituents of bone at the nanoscale are Type I collagen and hydroxyapatite, woven into a complex two-phase composite. Osteogenesis Imperfecta (OI) is a heritable disorder caused by mutations in collagen. The dominant OI phenotype is in bone and is typified by low energy fracture, leading to the term "brittle bone" disease. Normal collagen is a heterotrimeric molecule composed of alpha chains (two $\alpha 1$ and one $\alpha 2$ ) wound into a triple helix. Autosomal dominant OI Types II, III and IV result from point mutations causing the 
substitution of a triple-helical glycine (80\%) or splice site mutations (20\%) in the genes encoding the $\alpha$ chains [1]. Changes in $\alpha$ chain structure in Ol cause delayed protein folding, overmodification and decreased collagen quality [2].

Significant advances in OI research have been facilitated through the development of animal models. The Osteogenesis Imperfecta murine (oim) model was first described in 1993 [3]. This mouse has a single base pair mutation in the $\alpha 2$ gene that causes a frame shift of the final 48 amino acids at the C-terminus of the propeptide, changing the amino acid sequence and adding an extra residue. The resulting $\alpha 2$ chains cannot associate with $\alpha 1$ chains [4]. In the absence of functional $\alpha 2$ chains, homotrimeric $\alpha 1$ collagen molecules are formed which accumulate in the extracellular matrix and disrupt fibril assembly. While mutations in the $\alpha 2$ gene are rare in humans, the oim model closely matches human type III OI phenotypically, resulting in a moderate to severe OI phenotype characterized by spontaneous fractures and limb deformities [5].

Recent research has uncovered many abnormalities in both collagen and mineral in oim mice including altered mineral crystal size, heterogeneity and composition [6-14], changes in collagen content and cross-linking $[6-8,12,13]$ and abnormal collagen fibril structure $[15,16]$. Reduced bone size $[7,8,13,17,18]$ and generally brittle mechanical behavior at both the tissue and structural levels has also been observed [7, 10, 13, 14, 17, 19].

This wealth of studies noted above makes it clear that molecular changes in collagen are responsible for reduced mechanical properties associated with OI, but the link between these levels of tissue hierarchy remains elusive. Using new tissue characterization techniques, the goal of this study was to perform a multi-scale phenotypic analysis of bone from oim mice while maintaining samples as close to physiological conditions as possible. It was hypothesized that an inability to properly form and organize the collagen matrix impacts the formation and 
performance of the inorganic phase of bone, leading to brittle tissue behavior, reduced strength and altered structural organization.

\section{MATERIALS AND METHODS}

Male mice were obtained from The Jackson Laboratory at 12 weeks of age (B6C3Fe a/a-col1a2 oim/Col1a2 ${ }^{\text {oim }}$ (oim/oim, $\left.n=11\right)$; B6C3FeF1/J a/a $(W T, n=15)$ ). Animals were sacrificed, weighed, and femora were harvested and stored wrapped in phosphate buffered saline (PBS) soaked gauze at $-20^{\circ} \mathrm{C}$. All animal handling was done with prior IACUC approval (protocol number SC197R).

Right femora ( $n=5$ per group) were processed and imaged in air using atomic force microscopy (AFM) as previously described [20]. Images were acquired at 3 sites along the anterior length of the bone, and D-spacing of individual collagen fibrils was determined using a 2D Fast Fourier Transform ( -55 fibrils per bone).

Raman spectroscopy was performed as described previously [21]. Briefly, spectra were acquired at five locations approximately $1 \mathrm{~mm}$ apart along the posterior surface of fresh, unprocessed right femora ( $n=5-6$ per group: LabRAM HR 800 Raman Spectrometer). A 660 nm laser was focused on the sample surface using a 50X objective to a spot size of $\sim 10 \mu \mathrm{m}$. Bones were submerged in a PBS bath with the surface exposed. Five $20 \mathrm{sec}$ acquisitions were averaged at each location and baseline corrected to create a single measure at each location (LabSpec 5, HORIBA Jobin Yvon). Data from all locations were pooled into a single measurement for each metric from each bone for group comparisons ( $n=5-6$ per group).

Following Raman spectroscopy, the same femora were tested using Reference Point Indentation (RPI) (Active Life Scientific, Santa Barbara, CA). All bones were hydrated with PBS throughout indentation. Four indentations were made at approximately the same location as four of the five Raman measurements (limited to 4 locations due to orientation restrictions with the RPI fixture). Cyclic indentation was performed in load control to $2 \mathrm{~N}$ for 5 loading cycles. 
Measurements from the four locations in each bone were averaged to produce a single value for each metric in each bone for group comparisons.

Microcomputed tomography $(\mu \mathrm{CT})$ scans of left femora were performed at an isotropic voxel size of $12 \mu \mathrm{m}$ (Skyscan 1172, Bruker-MicroCT, Kontich, Belgium). Volumetric bone mineral density (VBMD) measures were made at five sites from the posterior quadrant of bone to complement Raman measures. Cortical geometry was measured from 7 slices centered at the midshaft. Trabecular architecture was measured from a $5 \mathrm{~mm}$ region of interest located just proximal to the distal growth plate.

Following $\mu \mathrm{CT}$, left femora were tested to failure in 3-point bending in displacement control (posterior surface in tension) at $0.3 \mathrm{~mm} / \mathrm{sec}$ using a $5 \mathrm{~mm}$ support span (Test Resources, Shakopee, MN). Force and displacement were measured during each test. The fracture location was measured from the distal end using calipers, then a subset of geometric properties at the fracture location was obtained from $\mu \mathrm{CT}$ to calculate stress and strain.

All data are presented as mean \pm standard deviation unless otherwise indicated. Statistical analysis was performed using two-sample T-tests or non-parametric Mann-Whitney $U$ test when appropriate. Kolmogorov-Smirnov (KS) tests were performed to assess population differences in collagen fibril D-spacing distributions. In all cases, a value of $p<0.05$ was considered significant.

\section{RESULTS}

AFM measurements from each bone were averaged to produce the mean fibril spacing for that bone. D-spacing in oim/oim $(65.4 \pm 0.5 \mathrm{~nm})$ was significantly lower than WT $(66.0 \pm 0.2$ $n m, p=0.008)$. Analysis of all fibrils within each group ( $n=289$ for $W T, n=254$ for oim/oim) indicated that the entire oim/oim population of fibrils was shifted downward versus WT towards lower spacing values $(\mathrm{p}<0.001$, Figure 1). The oim/oim population was, on average, $0.73 \mathrm{~nm}$ lower than WT. 


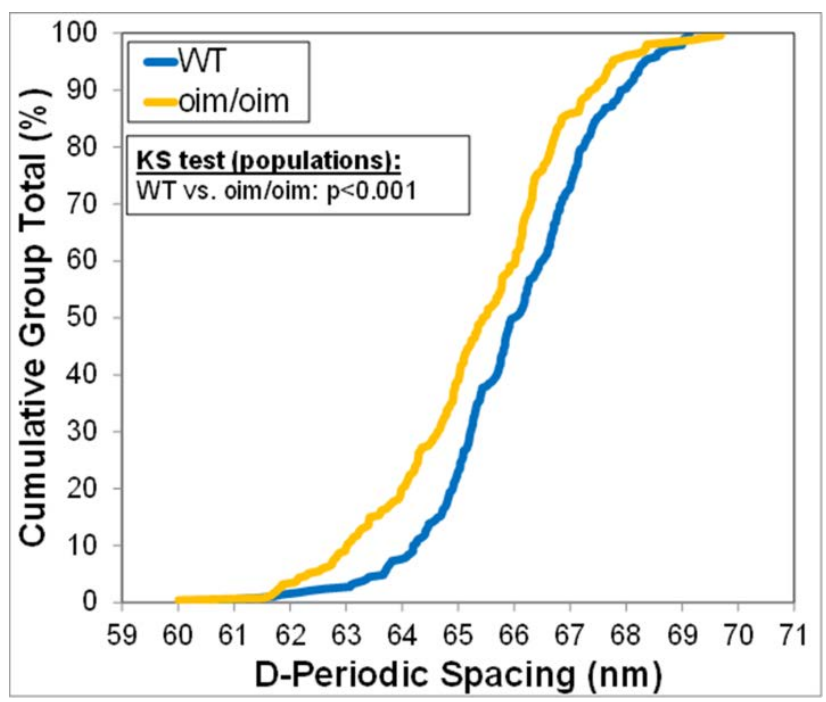

Figure 1: Cumulative Distribution Function (CDF) of all collagen fibrils measured in each group. The offset between the distributions points toward a significant downward shift in D-spacing in oim/oim versus WT.

All Raman measures of mineral-to-matrix ratio (MMR) showed a decreasing trend in oim/oim, but only reached significance for MMR Amide I ( $p=0.002$, Table S1). vBMD assessed via $\mu C T$ in similar regions of the bone was also marginally lower in oim/oim $(p=0.069)$. A significant decrease in mineral crystallinity accompanied decreased mineralization in oim/oim $(p<0.001)$. Amide I:Amide III was significantly increased in oim/oim suggesting a change in the secondary structure of collagen given the relative stability of Amide III and insensitivity to polarization effects versus Amide I ( $p=0.003)$. The $1660 / 1690 \mathrm{~cm}^{-1}$ intensity ratio was significantly decreased in the oim/oim group $(p=0.049)$, indicating a reduction in the maturity of collagen crosslinks.

RPI measures local tissue mechanical properties (Table S2). There was a significant increase in average indentation distance $(p=0.030)$ and significantly lower unloading slope for the first cycle $(p=0.002)$ versus WT. No other differences reached statistical significance, but total indentation distance was marginally higher in oim/oim $(p=0.051)$.

Across all cortical geometric properties measured, oim/oim bones were significantly smaller than WT with the exception of a marginal decrease in endocortical perimeter (schematic 
representation in Figure S3, data in Table S4). Similar to cortical bone changes, trabecular bone volume fraction and thickness were lower and trabecular spacing was higher in oim/oim $(p<0.001$ for all, Table S5).
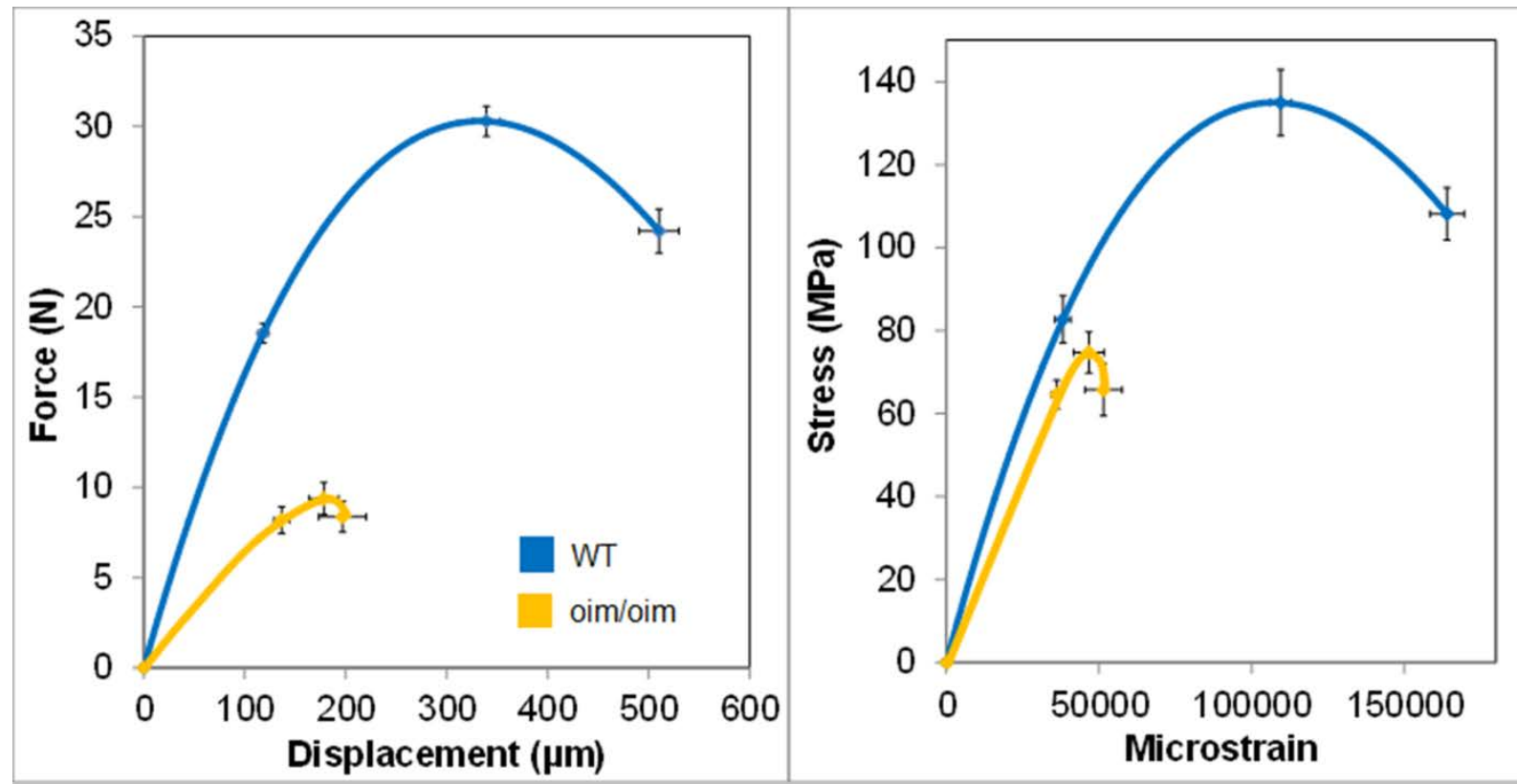

Figure 2: Average structural (left) and material (right) response to three-point bending. Oim/oim had significantly weaker and more brittle structural and tissue properties. Full dataset of measures shown in Table S6.

At the structural level, all mechanical properties were significantly decreased in oim/oim with the exception of increased elastic deformation (Table S6).When normalized to geometry, apparent tissue level properties showed similar trends (Figure 2). All properties trended downward and reached significance with the exception of modulus $(p=0.164)$ and elastic strain $(p=0.592)$.

\section{DISCUSSION}

As a genetic disease model that alters collagen formation, analyses in oim bone require studying nanoscale features to understand how a molecular change in collagen can alter material and structural-level properties in bone. Testing at multiple length scales provides insight into how changes in cellular activity and tropocollagen processing lead to alterations in morphology, tissue chemistry and material mechanical properties. 
Measurements of D-spacing obtained from AFM measurements provide structural information of collagen at the nanoscale. Mean differences in D-spacing between groups are not typical in disease states as distributions can be wide and sample numbers are typically small (i.e. $n=5)$. In this case, the significant mean difference was driven by a downward shift in the population of oim/oim D-spacing. This shift was likely caused by an overall decrease in tropocollagen persistence length leading to kinking and disorganization within the homotrimeric molecules [22]. This change may also lead to changes in the end-to-end distance of molecules and could alter the tightness of the microfibril twist [23]. Decreased D-spacing could provide less space for water and misalignment of crosslinking sites, impacting viscoelastic and post-yield behavior in the tissue. There could also be less space available for mineral nucleation and growth, indicating that a change in the organic portion is directly impacting the inorganic phase.

Altered chemical and matrix composition in oim/oim bone is apparent from Raman data. Reduced mineral crystallinity suggests a defect in the binding and growth of mineral crystals within the collagen matrix,_which along with the decreased D-spacing could explain the smaller crystal size noted in oim/oim here and elsewhere $[9,10]$. The trend toward reduced mineralization in MMR and VBMD implies there may be a slight reduction in mineralization, but this reduction was only significant when calculating MMR using the Amide I band. This reduction in MMR conflicts with previous studies that have analysed MMR using the same measure. Studies have reported mineralization to be unchanged [7] by FTIR in femora and increased [13] by Raman in tibiae, but both with significantly altered mineral composition as indicated by reduced Carbonate:Phosphate. These differences could be explained by a difference in technique performed. As opposed to analysis of cortical cross-sections or trabeculae which may include data from both mature and new bone growth, our periosteal measures provided results from a consistent penetration depth and likely a more homogeneous and young tissue age. This could also hold true for the contradictory levels of crosslink maturity shown in our data.[8] 
The Amide I band is more sensitive to polarization effects than other collagen bands [24] and the orientation of collagen in WT and oim/oim would increase the Amide I intensity [25]. However, samples from both groups were aligned in the same direction and the collagen orientation between WT and oim/oim mice are not statistically different [25] indicating that while individual spectra may vary due to polarization, the polarization effect does not explain the significant changes between groups. Therefore, while the increased Amide I:Amide III ratio may be partially explained by polarization, the differences between groups likely arises from the significant reduction in crosslinking maturity and the altered collagen structure noted from the AFM data.

$\mathrm{RPI}$ is an emerging technology that is capable of obtaining clinically-relevant microscale mechanical data while minimizing structural damage induced to the specimen [26]. This technique was coupled with Raman as the two techniques test a similar volume of material. Decreased unloading slope and increased indentation distance indicate a decreased ability of the oim/oim tissue to resist elastic and plastic deformation. Unloading slope serves as a representation of elastic stiffness as elastic deformation is the only recoverable portion of overall deformation. Increased indentation distance shows that the bone is brittle and less resistant to deformation. These changes may be microscale manifestations of the noted changes in mineral and collagen. However, most properties failed to correlate with Raman measures with the exception of crystallinity. Although the two techniques test the same volume of bone, Raman is measuring molecular level changes so length scale may still be an issue.

Across both structural-level and apparent tissue-level mechanical properties, the major functional impact of the oim mutation can be seen as almost all properties were decreased in oim/oim versus WT. Changes were noted in both elastic and post-yield portions of the curves, but post-yield behavior in the oim/oim bone was nearly absent, indicative of brittle behaviour. Changes in both pre-yield and post-yield behavior indicate that the genetic alteration in collagen is influencing both the organic and inorganic phases of the material, and possibly the 
relationship between collagen and mineral. In an attempt to understand the relationships between the various length scales, linear correlation analyses were undertaken. Of particular interest, both crystallinity and D-spacing were highly and significantly correlated with mechanical properties obtained from both whole bone tests and RPI. Because of a lack of overlapping samples between techniques, sample size was a limitation. Future studies will further investigate these relationships. Although data also indicate a cellular defect in the oim/oim mice leading to decreased bone mass, geometry and trabecular architecture, this phenomenon was not investigated here and will also be the topic of future research.

\section{CONCLUSIONS}

Overall, this study demonstrates how molecular changes to collagen due to a genetic defect can drastically alter properties throughout the tissue hierarchy in both the organic and inorganic portions of bone. Alterations to the collagen triple helix modify the assembly of collagen fibrils. We postulate that the fibrillar defect detected by AFM changes how hydroxyapatite crystals are able to bind and grow in this altered matrix. Changes in the collagen/mineral composite impact mechanical properties of the tissue and structure. Future studies will be aimed at assessing nonmineralized tissues to look for similar shifts in periodic Dspacing as well as changes in fibrillar width. These studies will allow for a direct analysis of collagen without the convoluting influence of mineral.

\section{REFERENCES}

[1] J. C. Marini, A. Forlino, W. A. Cabral, A. M. Barnes, J. D. San Antonio, S. Milgrom, et al., "Consortium for osteogenesis imperfecta mutations in the helical domain of type I collagen: regions rich in lethal mutations align with collagen binding sites for integrins and proteoglycans," Human mutation, vol. 28, pp. 209221, 2007.

[2] F. H. Glorieux, L. M. Ward, F. Rauch, L. Lalic, P. J. Roughley, and R. Travers, "Osteogenesis imperfecta type VI: a form of brittle bone disease with a mineralization defect," Journal of Bone and Mineral Research, vol. 17, pp. 30-38, 2002.

[3] S. D. Chipman, H. O. Sweet, D. J. McBride, M. T. Davisson, S. C. Marks, A. R. Shuldiner, et al., "Defective pro alpha 2 (I) collagen synthesis in a recessive 
mutation in mice: a model of human osteogenesis imperfecta," Proceedings of the National Academy of Sciences, vol. 90, pp. 1701-1705, 1993.

[4] D. J. McBride and J. R. Shapiro, "Confirmation of a G nucleotide deletion in the Cola-2 gene of mice with the osteogenesis imperfecta mutation," Genomics, vol. 20, pp. 135-137, 1994.

[5] D. O. Sillence, A. Senn, and D. Danks, "Genetic heterogeneity in osteogenesis imperfecta," Journal of medical genetics, vol. 16, pp. 101-116, 1979.

[6] N. P. Camacho, L. Hou, T. R. Toledano, W. A. Ilg, C. F. Brayton, C. L. Raggio, et al., "The material basis for reduced mechanical properties in oim mice bones," Journal of Bone and Mineral Research, vol. 14, pp. 264-272, 1999.

[7] N. Camacho, W. Landis, and A. Boskey, "Mineral changes in a mouse model of osteogenesis imperfecta detected by Fourier transform infrared microscopy," Connective tissue research, vol. 35, pp. 259-265, 1996.

[8] R. M. Coleman, L. Aguilera, L. Quinones, L. Lukashova, C. Poirier, and A. Boskey, "Comparison of bone tissue properties in mouse models with collagenous and non-collagenous genetic mutations using FTIRI," Bone, 2012.

[9] P. Fratzl, O. Paris, K. Klaushofer, and W. Landis, "Bone mineralization in an osteogenesis imperfecta mouse model studied by small-angle x-ray scattering," Journal of Clinical Investigation, vol. 97, p. 396, 1996.

[10] B. Grabner, W. Landis, P. Roschger, S. Rinnerthaler, H. Peterlik, K. Klaushofer, et al., "Age-and genotype-dependence of bone material properties in the osteogenesis imperfecta murine model (oim)," Bone, vol. 29, pp. 453-457, 2001.

[11] S. S. Mehta, P. P. Antich, and W. J. Landis, "Bone material elasticity in a murine model of osteogenesis imperfecta," Connective tissue research, vol. 40, pp. 189198, 1999.

[12] C. Phillips, D. Bradley, C. Schlotzhauer, M. Bergfeld, C. Libreros-Minotta, L. Gawenis, et al., " Oim mice exhibit altered femur and incisor mineral composition and decreased bone mineral density," Bone, vol. 27, pp. 219-226, 2000.

[13] X. Yao, S. M. Carleton, A. D. Kettle, J. Melander, C. L. Phillips, and Y. Wang, "Gender-Dependence of Bone Structure and Properties in Adult Osteogenesis Imperfecta Murine Model," Annals of Biomedical Engineering, pp. 1-11, 2013.

[14] M. Vanleene, A. Porter, P.-V. Guillot, A. Boyde, M. Oyen, and S. Shefelbine, "Ultra-structural defects cause low bone matrix stiffness despite high mineralization in osteogenesis imperfecta mice," Bone, vol. 50, pp. 1317-1323, 2012.

[15] D. J. McBride, V. Choe, J. R. Shapiro, and B. Brodsky, "Altered collagen structure in mouse tail tendon lacking the $\alpha 2$ (I) chain," Journal of molecular biology, vol. 270, pp. 275-284, 1997.

[16] K. Misof, W. Landis, K. Klaushofer, and P. Fratzl, "Collagen from the osteogenesis imperfecta mouse model (oim) shows reduced resistance against tensile stress," Journal of Clinical Investigation, vol. 100, p. 40, 1997.

[17] J. McBride, DJ, J. Shapiro, and M. Dunn, "Bone geometry and strength measurements in aging mice with the oim mutation," Calcified tissue international, vol. 62, pp. 172-176, 1998. 
[18] S. Rao, K. Evans, A. Oberbauer, and R. Martin, "Bisphosphonate treatment in the oim mouse model alters bone modeling during growth," Journal of biomechanics, vol. 41, pp. 3371-3376, 2008.

[19] E. Miller, D. Delos, T. Baldini, T. M. Wright, and N. P. Camacho, "Abnormal mineral-matrix interactions are a significant contributor to fragility in oim/oim bone," Calcified tissue international, vol. 81, pp. 206-214, 2007.

[20] J. M. Wallace, B. G. Orr, J. C. Marini, and M. M. B. Holl, "Nanoscale morphology of Type I collagen is altered in the Brtl mouse model of Osteogenesis Imperfecta," Journal of structural biology, vol. 173, pp. 146-152, 2011.

[21] M. A. Hammond, M. A. Gallant, D. B. Burr, and J. M. Wallace, "Nanoscale changes in collagen are reflected in physical and mechanical properties of bone at the microscale in diabetic rats," Bone, vol. 60, pp. 26-32, 2014.

[22] S.-W. Chang, S. J. Shefelbine, and M. J. Buehler, "Structural and mechanical differences between collagen homo-and heterotrimers: Relevance for the molecular origin of brittle bone disease," Biophysical Journal, vol. 102, pp. 640648, 2012.

[23] J. P. Orgel, T. C. Irving, A. Miller, and T. J. Wess, "Microfibrillar structure of type I collagen in situ," Proceedings of the National Academy of Sciences, vol. 103, pp. 9001-9005, 2006.

[24] A. J. Makowski, C. A. Patil, A. Mahadevan-Jansen, and J. S. Nyman, "Polarization control of Raman spectroscopy optimizes the assessment of bone tissue," Journal of biomedical optics, vol. 18, pp. 055005-055005, 2013.

[25] M. Raghavan, N. D. Sahar, R. H. Wilson, M.-A. Mycek, N. Pleshko, D. H. Kohn, et al., "Quantitative polarized Raman spectroscopy in highly turbid bone tissue," Journal of biomedical optics, vol. 15, pp. 037001-037001-7, 2010.

[26] A. Diez-Perez, R. Güerri, X. Nogues, E. Cáceres, M. J. Peña, L. Mellibovsky, et al., "Microindentation for in vivo measurement of bone tissue mechanical properties in humans," Journal of Bone and Mineral Research, vol. 25, pp. 18771885, 2010. 